This item was submitted to Loughborough's Research Repository by the author.

Items in Figshare are protected by copyright, with all rights reserved, unless otherwise indicated.

\title{
Impact of non-synchronous generation on transmission oscillations paths
}

PLEASE CITE THE PUBLISHED VERSION

https://doi.org/10.1109/TPEC.2018.8312100

\section{PUBLISHER}

(C) Institute of Electrical and Electronics Engineers (IEEE)

\section{VERSION}

AM (Accepted Manuscript)

\section{LICENCE}

CC BY-NC-ND 4.0

\section{REPOSITORY RECORD}

Chamorro, Harold R., Camilo A. Ordonez, Jimmy C-H. Peng, Francisco M. Gonzalez-Longatt, Vijay K. Sood, and Al-Hossein M. Sharaf. 2019. "Impact of Non-synchronous Generation on Transmission Oscillations Paths". figshare. https://hdl.handle.net/2134/32389. 


\title{
Impact of Non-Synchronous Generation on Transmission Oscillations Paths
}

\author{
Harold R. Chamorro, Member, IEEE, Camilo A. Ordonez, Member, IEEE, \\ Jimmy C-H. Peng, Member, IEEE, Francisco Gonzalez-Longatt, Senior Member, IEEE, and Vijay K. Sood, Senior \\ Member, IEEE and Adel M. Sharaf, Senior Member, IEEE
}

\begin{abstract}
The large scale penetration of non-synchronous generation has been causing several impacts on the power systems dynamics. The low-frequency oscillations affect the power exchanged along the transmission lines/corridors. This paper uses the Multi-Prony Analysis mode estimation technique to monitor and suggest the dominant oscillation modes which can be useful for wide-area control purposes. Moreover, the oscillation modes are also monitored under gradual cases of non-synchronous generation integration in the system. The methodology is applied to two different test transmission systems: i) the two area system and, ii) the Nordic 32 system. The results illustrate the similarity and differences in the scenarios proposed.
\end{abstract}

Index Terms-Non-Synchronous Generation, Transmission Corridors, Oscillations Paths, Prony Analysis, Moving Window, System Inertia, Wide Area Measurements

\section{INTRODUCTION}

$\mathbf{R}$ ENEWABLE power has seen dramatic expansion in recent years due to the global governamental initiatives that looks for the reduction of Green House Gas (GHG) emissions caused by the burning of fossil fuels. But this growth has raised several challenges for power-system operators and energy policy regulators.

Systematic measures for integrating variable renewable power is a priority for many countries where the share of renewable power is growing rapdily. For instance, in nordic countries, variable renewable power is becoming an important component in the pool source of power, and therefore a comprenhensive study of the power system is necessary.

The composition of Wide Area Measurement Systems (WAMS) are based on operational tools that are continuously monitoring various signals (e.g., voltages, currents, speed of machines) in the grid. The inclusion of distributed Phasor Measurement Units (PMUs) in the system has allowed to

Harold R. Chamorro is a PhD student at KTH Royal Institute of Technology, Stockholm, Sweden, e-mail: hr.chamo@ieee.org, hrcv@kth.se.

Camilo Ordonez is Engineer at Planning and New Bussiness Departmen Transmission Vicepresidency in Bogota Electrical Company (EEB), e-mail: c.a.ordonez@ieee.org.

Jimmy C. Peng is with the Department of Electrical and Compute Engineering of National University of Singapore. e-mail elepcj@nus.edu.sg.

Francisco Gonzalez-Longatt is with Loughborough University, Leicestershire, United Kingdom, e-mail: f.gonzalez-longatt@lboro.ac.uk

Vijay K. Sood is with the Department of Electrical and Computer Engineering of University of Ontario, Institute of Technology, Ontario, Canada e-mail: vijay.sood@uoit.ca.

Adel M. Sharaf is with Sharaf Energy Systems, New Brunswick, Canada e-mail: proddramsgaraf@yahoo.ca. transmit, process and comprehend these signals in order to improve and protect the system to undersirable events [1]

One of the objectives of WAMS is to identify/estimate dominant low-frequency oscillations modes along the power grid and, in real-time, activate the alarms, protection systems or controllers involved in the oscillations monitoring and restoration process (e.g., Power System Stabilizers) [2].

Additionally, with the current expansion of nonsynchronous generation interconnection on conventional generation (synchronous generators), several power planners and researchers, have reported significant dynamical challenges during the past years [3]. One of the aspects which can be adversely affected by the inclusion of nonsynchronous generation is the oscillatory stability [4]. Hence, low frequency electromechanical oscillations deteriorate the power transfer exchanges, degrade the power system security, and ocasionally, provoke cascading failures or even provoke a system blackout [5]. Therefore, it is important to understand how these oscillations vary under non-synchronous generation inclusion.

Several studies have shown that the large scale inclusion of wind power degrades the damping of the electromechanical modes. This displaces them to a different stability operation region, thereby affecting the response of the system under small and large disturbances [6].

Signal processing and spectral decomposition methods play a key role for the oscillation estimation methods in power systems based on measurements. The main methods applied to this topic include Hankel Singular Value Decomposition (HSVD) [7], Hilbert Spectral Analysis [8], the Extended Complex Kalman Filter (ECKF) [9], and wavelet-based method [10], [11].

Another method which has been used extensively for the estimation of oscillatory modes is the Prony analysis [12]. In [13], Prony Analysis (PA) method is used to detect poorly damped oscillations in early stages of the disturbance based on a size-controlled sliding window. In a recent contribution [14], and tested in a benchmark power system , PA method has been improved and used for extracting the dominant modes including a shrinking window.

The oscillation tracking paths along power systems have been studied in [15] and [16], however the only research proposal tracking oscillations including renewables is presented in [17], therefore the motivation of this paper is to analyse the 
oscillation paths under gradual inclusion of non-synchronous generation.

A preliminary version of this work has been published in [18]. This work contains substantial improvements to the proposed study and new simulations that do not appear in [18]. In contrast to [18], this paper focuses on tracking the oscillations along the entire system in order to recognise the oscillations path. Moreover different scenarios for tracking the signals are presented. Multi-Prony Analysis (MPA) mehtod also uses a sliding window with the purpose of emulate the PMU sampling and to have a better understanding of the propagation of the oscillations in the system through graphical observation. Furthermore, it is demonstrated that the proposed study can be used as a monitoring tool due to it is based on the measurement of the system. This paper, analyses the effect of gradually increasing replacement of synchronous generators by high penetration of aggregated non-synchronous generation on power systems, including full converters as an interface on the transmission oscillations paths applying the MPA.

The paper is structured as follows: Section II presents briefly the concept of the method developed. In Section III, the oscillation paths tracking concept is presented. Section IV presents the Prony theory and the on-line oscillations path method explaied. In section $\mathrm{V}$, the method is evaluated in two tests systems. Also, the case studies regarding the gradual increasing of non-synchronous generation integration in one of the test systems is presented. Finally, the conclusions and future work of this research are given.

\section{OSCILlations PATHS TRACKING CONCEPT}

Monitoring of on-line mode estimation can be achieved by installing PMUs in the main tie-lines, so the active power measurement can be obtained and processed in the control center. Then, special algorithms, like the one presented in this paper, can determine and track the modes, and detect a possible cascading effect, separation of areas, loss of synchronism between machines during early stages of post-contingency state. Therefore, monitoring on-line real-time oscillation paths can straightforwardly distinguist between prevention or not of a partial or total blackout [19].

Figure 1 shows the concept of the on-line oscillations tracking along a signal. After a disturbance, an oscillation is provoked. The signal, as in this case, can be composed of one, two or more oscillatory modes at different frequencies and different damping ratios. As it can be seen in the figure, two sets of data $Y_{1}$ and $Y_{2}$ are deconstructed by the moving window algorithm based on PA.

\section{NON-SYNCHRONOUS GENERATION}

In this paper, non-synchronous generation is defined as when the power is provided/absorbed through power electronic converters (DC-AC) to the system. Voltage Source Converters (VSC) have been used for wind power turbines namely, Full Rated Converters (FRC), Doubly-Fed Induction Generator (DFIG), and High Voltage Direct Current (HVDC) and Multiterminal HVDC connection [20].

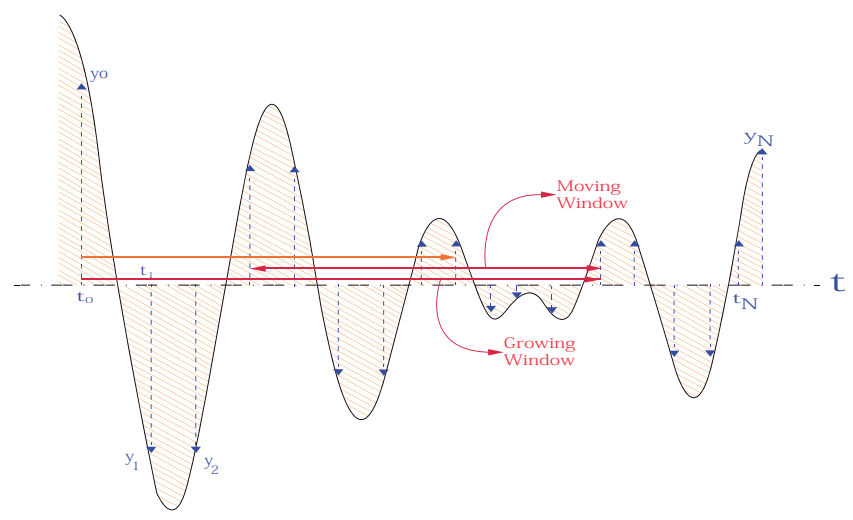

Fig. 1. Moving Window Concept

The non-synchronous generation allows to control active and reactive power control, however the generation is completely decoupled from the system, and is consequently unable to contribute dynamically to the system. Thus, if the synthetic inertia control option is not added, the decoupling displaces synchronous machines [21], [22].

\section{Prony Analysis And On-Line Coherency}

Prony analysis is a least-square approximation technique for fitting a sum of exponential terms to the measured data. It then identifies the amplitudes, damping factors, frequencies and phases contained inside the observed signal. For power systems, these estimations are conducted under the assumption that the system has encountered a perturbation set up following line or generator outages [23].

Consider a general continous signal written as:

$$
y(t)=\sum_{i=1}^{m} R_{i} e^{\lambda_{i} t}
$$

where $R_{i}$ are the residues and are directly linked with the amplitude of each mode to form the signal. When it is sampled at a constant sampling period $\Delta t$, the following expression represent the sampled signal:

$$
y(k)=\sum_{i=1}^{m} R_{i} z_{i}^{k}
$$

where $k$ are the samples, and $z_{i}=e^{\lambda_{i} \Delta t}$, with $\lambda=\sigma+i \varpi$. Since the real power system is not purely linear, this is an approximation of the adjustment of the measured signals with the aim of identifying the damping of the modes involved in the variables. Damping ratio is defined as the real part of the mode divided by its magnitude. It can be expressed as a percentage.

Prony methodology is used to estimate the modes of oscillation of a signal, identifying the relative contribution of each mode to form the signal, by the computation of the residues. Rewriting (2) in matrix form:

$$
\left[\begin{array}{c}
y(0) \\
y(1) \\
\cdots \\
y(N-1)
\end{array}\right]=\left[\begin{array}{cccc}
1 & 1 & \cdots & 1 \\
z_{1} & z_{2} & \cdots & z_{n} \\
\cdots & \cdots & \cdots & \cdots \\
z_{1}^{N-1} & z_{2}^{N-1} & \cdots & z_{n}^{N-1}
\end{array}\right]\left[\begin{array}{c}
R_{1} \\
R_{2} \\
\cdots \\
R_{n}
\end{array}\right]
$$


where $N$ is the number of samples. Additionally, $z_{i}$ satisfies a polynomial of order $n$ with the coefficients being $a_{i}$ whose $n$ roots are the modes of the group of signals:

$$
z^{n}-\left(a_{1} z^{n-1}+a_{2} z^{n-2}+\cdots+a_{n} z^{0}\right)=0
$$

Multiplying the vector $\left[-a_{n},-a_{n-1}, \ldots, a_{1}, 1,0, \ldots, 0\right]$, to each side of (3), it is obtained that each sample may be written as a linear combination of the previous data and the coefficients are $a_{i}$, that is: $y(n)=a_{n} y(0)+a_{n-1} y(1)+\cdots+$ $a_{1} y(n-1)$. This can be written for each sample in a matrix form:

$\left[\begin{array}{c}y(n) \\ y(n+1) \\ \cdots \\ y(N-1)\end{array}\right]=\left[\begin{array}{cccc}y(n-1) & y(n-2) & \cdots & y(0) \\ y(n) & y(n-1) & \cdots & y(1) \\ \cdots & \cdots & \cdots & \cdots \\ y(N-2) & y(N-3) & \cdots & y(N-n-1)\end{array}\right]\left[\begin{array}{c}a_{1} \\ a_{2} \\ \cdots \\ a_{n}\end{array}\right]$

The methodology of Prony can be summarized in three steps:

1. Solve (5) to get the coefficients $a_{i}$.

2. Next, obtain the roots of the polynomial in (4). In this step, it is now possible to get the modes: $\lambda_{i}=\sigma+i \omega=$ $\operatorname{Ln}\left(z_{i}\right) / \Delta t$.

3. The third step is to get the complex residues $R_{i}$ solving (3), by using the solutions of (4). The time between samples from PMU is $\Delta t=33 \mathrm{~ms}$. This value is assumed for the subsequent simulations.

\section{Study Cases and Application}

In order to illustrate the method, two test power systems are proposed as follows:

\section{A. Case 1}

The single line diagram of the test case 1 is shown in Figure 2. The test system consists of two fully symmetrical areas linked together by two $230 \mathrm{kV}$ lines of $220 \mathrm{~km}$ length. Each area is equipped with two identical round rotor generators rated $20 \mathrm{kV} / 900 \mathrm{MVA}$. The nominal power system frequency for the test case model is $50 \mathrm{~Hz}$. It was specifically designed in [24] to study low frequency electromechanical oscillations in large interconnected power systems. The loads are represented as constant impedances and split between the areas in such a way that area 1 is exporting power to area 2 . In order to analyze the response of the power system, a large disturbance in the form of three phase to ground fault ( 4 cycles i.e. 80 ms) at $t=2 \mathrm{~s}$ is introduced in the middle of one of the two $220 \mathrm{~km}$ transmission line connecting Area 1 with Area 2. This results in an undamped oscillation of $0.6 \mathrm{~Hz}$.

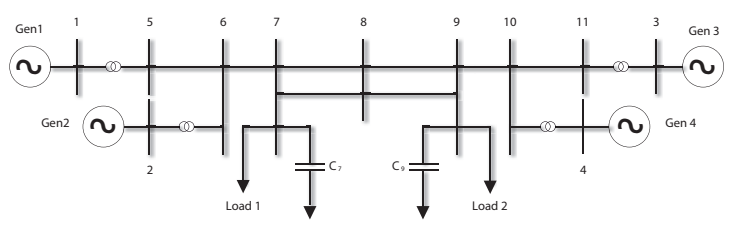

Fig. 2. Two Area Test System
1) On-line Prony: From the measurements of the active power through the tie-lines (Figure 3), it is plotted the tracked oscillations based on the on-line PA algorithm proposed. As it can be seen, the samples tracked show a cluster of oscillations around $0.6 \mathrm{~Hz}$, which confirms the expected frequency oscillation in the system. Besides, it is plotted the decay factor which shows how the oscillations decay while the oscillations get diminished.

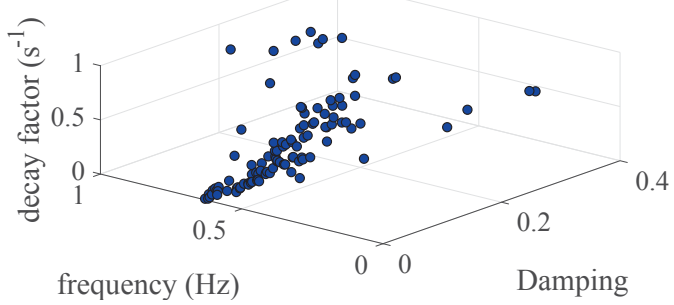

Fig. 3. On-line Frequency Dominant Frequency Modes Estimation

\section{B. Nordic System}

Figure 4 shows the single-line diagram of the Nordic test system presented in [25]. This system contains 32 high voltage buses. The transmission system is designed for $400 \mathrm{kV}$ with some regional systems at $220 \mathrm{kV}$ and $130 \mathrm{kV}$. This system has three identified areas of operation: North, Central, South and External. The simulation of this test system has been performed by the software DigSILENT ${ }^{\circledR}$. The dominant interarea mode frequency of this system is $0.56 \mathrm{~Hz}$ (this mode is also reported in the literature [26]).

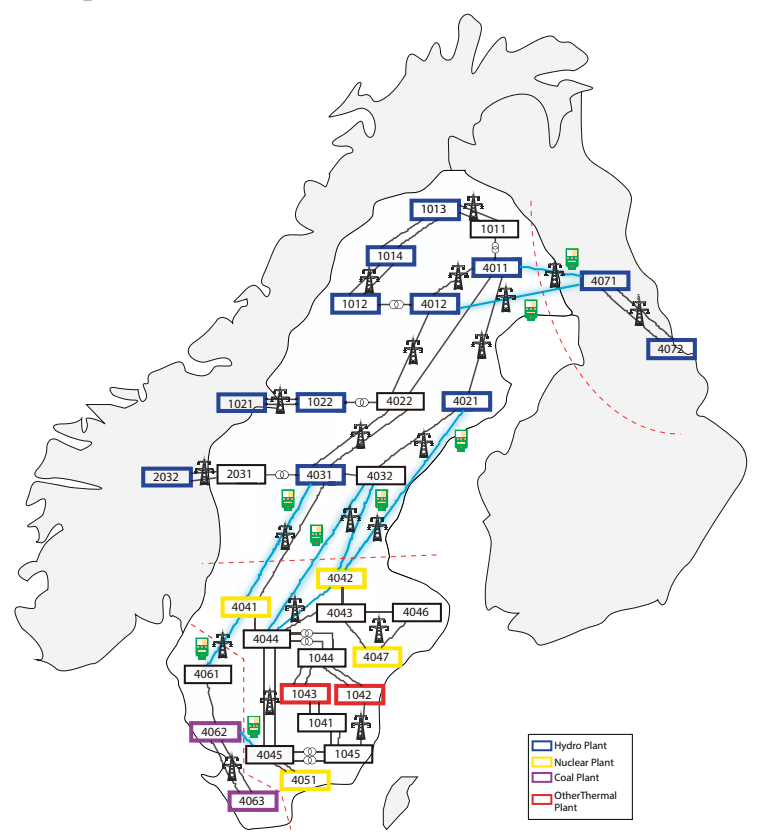

Fig. 4. Nordic Test System Representation

\section{Non-synchronous generation}

The impact of the integration of non-synchronous generation on the test system oscillations is analysed by replacing some of the synchronous generators with non-synchronous 
generation with the same active and reactive power outputs. Note that the power outputs are fixed through the simulation. Case $1\left(C_{1}\right)$ to Case $5\left(C_{5}\right)$ represent the replacement of synchronous generation by the integration of non-synchronous generation gradually, in order to analyse different levels of power penetration. For example $C_{1}$ considers the replacement of one generation only, and $C_{2}$ considers the replacement of two generators including the one in $C_{1}$, and so on. It is assumed that the dispersed generation is connected to one established substation. These five scenarios are summarised in Table I. However, only the cases where it has been found significant changes are selected to be presented.

TABLE I

Generator Replacement For EACH CASE

\begin{tabular}{|c|c|c|c|c|c|}
\hline Case & 1 & 2 & 3 & 4 & 5 \\
\hline \hline Generator & $G_{7}$ & $G_{16}$ & $G_{17}$ & $G_{14}$ & $G_{6}$ \\
\hline Bus & 1043 & 4051 & 4062 & 4042 & 1042 \\
\hline Power (MW)\% & 1.2 & 5.4 & 9 & 13 & 15 \\
\hline
\end{tabular}

1) On-line Prony Application: The method is applied to the obtained measurements from the cases mentioned above and from the tie-lines shown in 4 . The tie-lines measurements are located in the operative areas interconnected as follows: South-Central (4062 - 4045, 4061 - 4041), Central-North (4021 - 4042, 4032 - 4042,4032 - 4044,4031 - 4041), NorthExt $(4071-4011,4071-4012)$. In order to observe the frequency similiraties, it is plotted the oscillations along the tie-lines mentioned.

Figure 5 shows two identified frequency sub-clusters for the base case. First cluster frequency mode is around $0.6 \mathrm{~Hz}$, and the second one is around $0.8 \mathrm{~Hz}$. Figures 6 to 9 (cases $C_{1}$ to $C_{5}$ ) show the frequency clusters obtained after the gradual inclusion of non-synchronous generation. Cases $C_{1}$ and $C_{2}$ do not show a particular change in the frequency modes, but after case $C_{3}$, the dominant mode moves to $0.4 \mathrm{~Hz}$ and the second mode moves out of the range of the electromechanical oscillations $([0.2,0.8]) \mathrm{Hz}$. Case $C_{4}$ and $C_{5}$ show to be the most drastically change in the dominant mode with a new frequency cluster is around $0.3 \mathrm{~Hz}$. This lattest frequency is related to the low-pass filters of the converters at the Point Common Coupling (PCC).

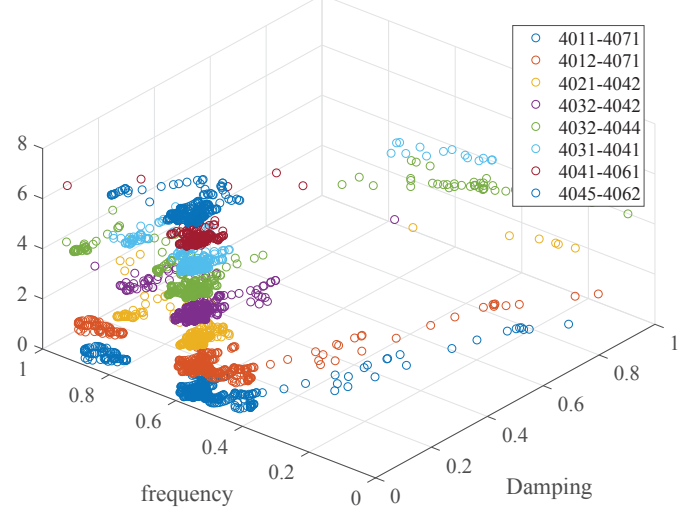

Fig. 5. Base Case

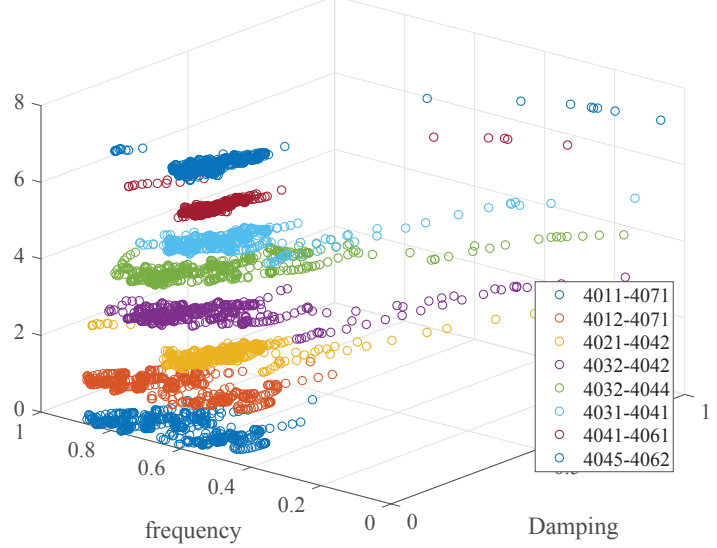

Fig. 6. Case 1

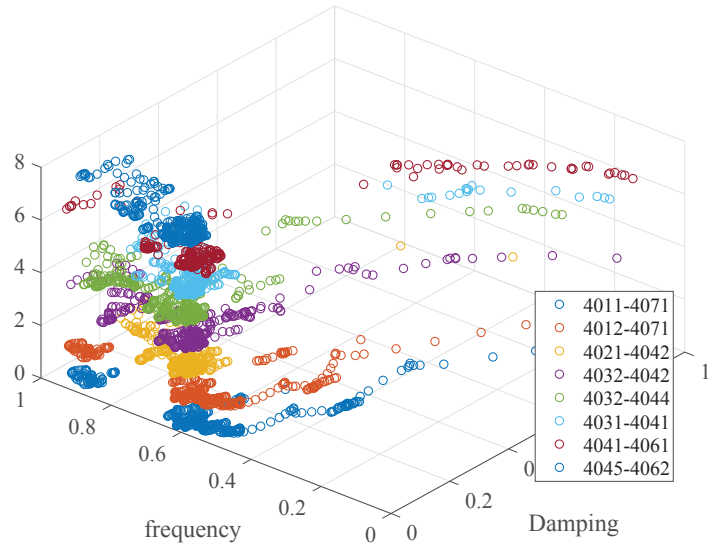

Fig. 7. Case 2

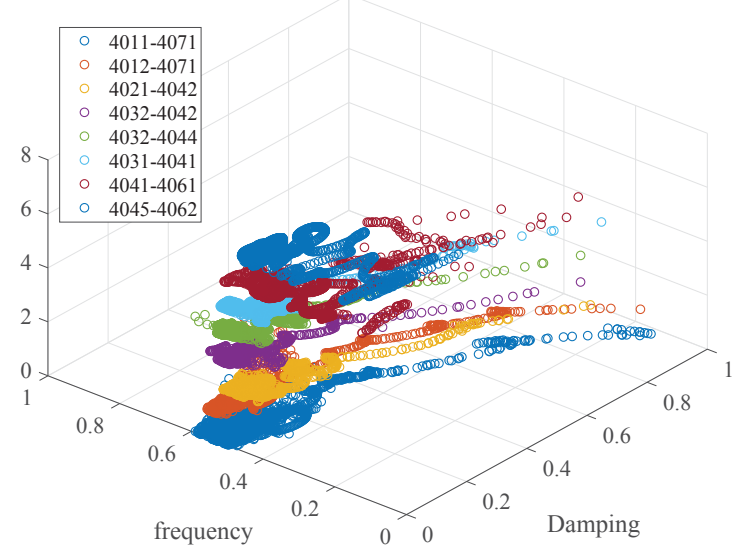

Fig. 8. Case 3

\section{CONCLUSIONS}

This document has presented the application of the on-line PA for tracking the oscillations along the tie-lines in the power system. By this method is possible to observe the oscillation frequency groups and the decay rates. The emulation of PMU 


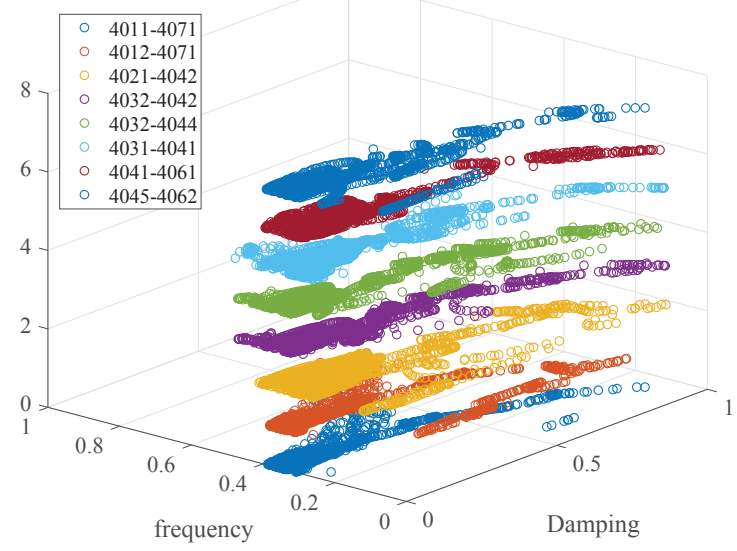

Fig. 9. Case 4 and 5

sampling provides information enough to track the oscillations, and observe their behaviour in the system.

By locating the measurements in the tie-lines the data processing can be reduced; however the location of the measurements depends on the topology of the system. Therefore, it is required more studies in this area.

The gradual increasing of non-synchronous generation drastically change the oscillations frequencies in the system. These changes might require to retune the PSS in the system, or change the location of the controllers in the system in order to do damp the oscillations adequately.

\section{REFERENCES}

[1] H. Hooshyar, L. Vanfretti, F. Mahmood, R. S. Singh, N. Singh, A. Bidadfar, and S. R. Firouzi, "Synchrophasor applications facilitating interactions in transmission and distribution operations," in 2017 IEEE Manchester PowerTech, Jun. 2017, pp. 1-6.

[2] D. Yang, W. Tang, C. Rehatanz, and K. Gorner, "A systematic method and its near-real time application to analyze the Low Frequency Oscillation based on WAMS," in 2013 Australasian Universities Power Engineering Conference (AUPEC), Sep. 2013, pp. 1-8.

[3] S.-H. Huang, D. Maggio, K. McIntyre, V. Betanabhatla, J. Dumas, an J. Adams, "Impact of wind generation on system operations in the deregulated environment: ERCOT experience," in IEEE Power Energy Society General Meeting, 2009. PES '09, Jul. 2009, pp. 1-8.

[4] H. R. Chamorro, M. Ghandhari, and R. Eriksson, "Influence of the increasing non-synchronous generation on Small Signal Stability," in 2014 IEEE PES General Meeting | Conference Exposition, Jul. 2014, pp. 1-5.

[5] Y. Hou, S. Mei, H. Zhou, and J. Zhong, "Blackout prevention: Managing complexity with technology," in 2008 IEEE Power and Energy Society General Meeting - Conversion and Delivery of Electrical Energy in the 21st Century, Jul. 2008, pp. 1-6.

[6] J. Quintero, V. Vittal, G. T. Heydt, and H. Zhang, "The Impact of Increased Penetration of Converter Control-Based Generators on Power System Modes of Oscillation," IEEE Transactions on Power Systems, vol. 29, no. 5, pp. 2248-2256, Sep. 2014.

[7] L. Di Noia, D. Lauria, C. Pisani, and D. Villacci, "A novel procedure for real-time detection of inter-area oscillations," in Energy Conference and Exhibition (ENERGYCON), 2012 IEEE International, Sep. 2012, pp. $433-438$.

[8] A. Q. Zhang, L. L. Zhang, M. S. Li, and Q. H. Wu, "Identification of Dominant Low Frequency Oscillation Modes Based on Blind Source Separation," IEEE Transactions on Power Systems, vol. 32, no. 6, pp. 4774-4782, Nov. 2017.

[9] J. C. H. Peng, N. Nair, J. Zhang, and A. Swain, "Detection of lightly damped inter-area power oscillations using extended complex kalman filter," in TENCON 2009 - 2009 IEEE Region 10 Conference, Jan. 2009 pp. 1-5.
[10] X. Pan and V. Venkatasubramanian, "Multi-dimensional wavelet analysis for power system oscillation monitoring using synchrophasors," in Innovative Smart Grid Technologies (ISGT), 2012 IEEE PES, Jan. 2012, pp. $1-10$.

[11] S. A. Hosseini, N. Amjady, and M. H. Velayati, "A Fourier Based Wavelet Approach Using Heisenberg's Uncertainty Principle and Shannon's Entropy Criterion to Monitor Power System Small Signal Oscillations," IEEE Transactions on Power Systems, vol. 30, no. 6, pp. 3314-3326, Nov. 2015.

[12] J. Hauer, C. Demeure, and L. Scharf, "Initial results in prony analysis of power system response signals," IEEE Transactions on Power Systems, vol. 5, no. 1, pp. 80-89, Feb. 1990.

[13] C. A. Ordóñez and M. A. Ríos, "Electromechanical modes identification based on sliding-window data from a wide-area monitoring system," Electric Power Components and Systems, vol. 41, no. 13, pp. 12641279, 2013. [Online]. Available: http://dx.doi.org/10.1080/15325008. 2013.816982

[14] D. P. Wadduwage, U. D. Annakkage, and K. Narendra, "Identification of dominant low-frequency modes in ring-down oscillations using multiple Prony models," Transmission Distribution IET Generation, vol. 9, no. 15, pp. 2206-2214, 2015.

[15] Y. Chompoobutrgool and L. Vanfretti, "Identification of Power System Dominant Inter-Area Oscillation Paths," IEEE Transactions on Power Systems, vol. 28, no. 3, pp. 2798-2807, Aug. 2013.

[16] H. M. Khalid and J. C. H. Peng, "Tracking Electromechanical Oscillations: An Enhanced Maximum-Likelihood Based Approach," IEEE Transactions on Power Systems, vol. 31, no. 3, pp. 1799-1808, May 2016.

[17] V. Terzija, D. Cai, and J. Fitch, "Monitoring of inter-area oscillations in power systems with renewable energy resources using prony method," in 20th International Conference and Exhibition on Electricity Distribution - Part 1, 2009. CIRED 2009, Jun. 2009, pp. 1-4.

[18] H. R. Chamorro, C. A. Ordonez, J. C. H. Peng, and M. Ghandhari, "Online oscillations monitoring under high penetration of non-synchronous generation," in 2015 IEEE International Conference on Smart Grid Communications (SmartGridComm), Nov. 2015, pp. 278-282.

[19] G. Zweigle, R. Moxley, B. Flerchinger, and J. Needs, "Wide-area measurements to improve system models and system operation," in 11th IET International Conference on Developments in Power Systems Protection (DPSP 2012), Apr. 2012, pp. 1-5.

[20] H. R. Chamorro, C. A. Ordonez, J. C. Peng, and M. Ghandhari, "Non-synchronous generation impact on power systems coherency," Transmission Distribution IET Generation, vol. 10, no. 10, pp. $2443-$ 2453, 2016.

[21] D. Banham-Hall, C. Smith, G. Taylor, and M. Irving, "Investigating the limits to inertial emulation with large-scale wind turbines with direct-drive permanent magnet generators," in UKACC International Conference on Control 2010, Sep. 2010, pp. 1-6.

[22] H. R. Chamorro, A. C. Sanchez, A. Øverjordet, F. Jimenez, F. GonzalezLongatt, and V. K. Sood, "Distributed synthetic inertia control in power systems," in 2017 International Conference on ENERGY and ENVIRONMENT (CIEM), Oct. 2017, pp. 74-78.

[23] J.-H. Peng and N.-K. Nair, "Adaptive sampling scheme for monitoring oscillations using prony analysis," IET Generation, Transmission Distribution, vol. 3, no. 12, pp. 1052-1060, Dec. 2009.

[24] M. Klein, G. Rogers, and P. Kundur, "A fundamental study of inter-area oscillations in power systems," IEEE Transactions on Power Systems, vol. 6, no. 3, pp. 914-921, Aug. 1991.

[25] C. T. Force, "Long term dynamics phase II final report." CIGRE, Mar. 1995.

[26] L. Vanfretti, S. Bengtsson, V. S. Perić, and J. O. Gjerde, "Spectral estimation of low-frequency oscillations in the Nordic grid using ambient synchrophasor data under the presence of forced oscillations," in 2013 IEEE Grenoble Conference, Jun. 2013, pp. 1-6. 